METZLER

KUNSTHISTORIKER LEXIKON 


\section{METZLER \\ KUNSTHISTORIKER LEXIKON}

Zweihundert Porträts deutschsprachiger Autoren aus vier Jahrhunderten

Von Peter Betthausen, Peter H. Feist und Christiane Fork unter Mitarbeit von Karin Rührdanz und Jürgen Zimmer 


\title{
Inhaltsverzeichnis
}

\author{
Vorwort V \\ Artikel und Autoren VIII \\ Abkürzungen (Orte; Zeitschriften, Periodika, Sammelbände; \\ Sonstiges; Autoren) XI \\ Artikel A-Z I \\ Bibliographie 501 \\ Namenregister 5I I
}

Die Deutsche Bibliothek - CIP-Einheitsaufnahme

Metzler-Kunsthistoriker-Lexikon : zweihundert Porträts

deutschsprachiger Autoren aus vier Jahrhunderten / von Peter

Betthausen ... - Stuttgart ; Weimar : Metzler, I999

ISBN $978-3-476$-oI $535-8$

ISBN $978-3-476-03698-8$ (eBook)

DOI $10.1007 / 978-3-476-03698-8$

Dieses Werk einschließlich aller seiner Teile ist urheberrechtlich geschützt.

Jede Verwertung außerhalb der engen Grenzen des Urheberrechtsgesetzes ist ohne Zustimmung des Verlages unzulässig und strafbar. Das gilt insbesondere für Vervielfältigungen, Übersetzungen, Mikroverfilmungen und die Einspeicherung und Verarbeitung in elektronischen Systemen.

(C) I 999 Springer-Verlag GmbH Deutschland

Ursprünglich erschienen bei J. B. Metzlersche Verlagsbuchhandlung

und Carl Ernst Poeschel Verlag GmbH in Stuttgart 1999 


\section{Vorwort}

Wer sich mit Kunstgeschichte beschäftigt, wird sehr oft nicht nur nach der jeweils neuesten Fachliteratur greifen, sondern zum Vergleich auch ältere Publikationen heranziehen. Die unterschiedlichen Urteile über ein Kunstwerk, die einander ablösten, gehören untrennbar zu dessen heutigem Verständnis, und jedes neue Erklären des Verlaufs der Kunstgeschichte hat immer bestimmte ältere Erklärungsvorschläge zur Voraussetzung.

Das Kunsthistorikerlexikon möchte - auch durch umfängliche Literaturnachweise über Leben und Leistungen von Kunstforschern informieren und dadurch zum Verständnis der Wissenschaftsgeschichte beitragen, indem es zeigt, wie die wechselnden, aufeinander Bezug nehmenden und gelegentlich sogar überraschend wiederauflebenden kunst- und kunstgeschichtstheoretischen Grundsätze und Methoden wissenschaftlicher Arbeit auch in den biographischen Bedingungen ihrer Urheber oderVerfechter, in bestimmten kultur- und geistesgeschichtlichen Situationen verankert waren.

Dafür wurden zweihundert Personen ausgewählt, die das Erforschen, Bewahren, Sammeln, Verstehen und Verbreiten von bildender Kunst, eingeschlossen die Architektur und die sogenannte angewandte Kunst, folgenreich vorangebracht oder in einer furr ihre Zeit typischen Weise betrieben haben, und deren Lebenswerk abgeschlossen vorliegt. Die Auswahl reicht von den Vorstufen einer im strengeren Sinne wissenschaftlichen Beschäftigung mit Kunstgeschichte über die einzelnen Etappen der Herausbildung einer selbständigen Kunstgeschichtswissenschaft bis in unsere Tage. Um einen gegebenen Gesamtumfang des Buches nicht zu sprengen, die einzelnen "Porträts" möglichst lesbar gestalten zu können, und außerdem Raum für ausführliche Literaturhinweise zu haben, beschränkt sich diese Auswahl auf deutschsprachige Autoren, einschließlich jener, die vom NS-Regime in die Emigration getrieben wurden und in anderen Sprachen weiterveröffentlichen mußten. Trotz des inzwischen weitestgehend internationalen Charakters der Kunstwissenschaft läßt sich diese Eingrenzung disziplingeschichtlich rechtfertigen. In vielen Jahrzehnten nach dem internationalen "Ersten kunstwissenschaftlichen Congress in Wien I 873 spielten Gelehrte aus deutschen, österreichischen und schweizerischen Museen und Universitäten, nächst denen aus Italien und Frankreich, eine allerseits anerkannte, maßgebliche Rolle sowohl in der Sachforschung, wie ganz besonders in der Methodenentwicklung.

Bei der Entscheidung, welche Personen aufzunehmen seien, wurde bedacht, daß die verschiedenen Berufszweige und speziellen Arbeitsfelder vertreten sein sollten, die sich im Verlauf der Zeit herausbildeten, und daß neben den unstrittigen Größen des Faches auch einige weniger Bekannte besser ins Licht gerückt werden sollten. Bei Universitätslehrern, Museumsmitarbeitern und Denkmalpflegern wie Kunstpublizisten und Kunstkritikern wurden diejenigen bevorzugt, die nicht nur ein Spezialgebiet erfolgreich bearbeiteten und die täglichen Aufgaben verdienstvoll 
erfullten, sondern den Blick auf weitere Zusammenhänge der Kunstentwicklung oder neue Gegenstände richteten und die Prinzipien und Methoden der fachlichen Arbeit bewuBt reflektierten und zu erneuern strebten. Ästhetiker fanden nur dann Aufnahme, wenn sie sich ausdrücklich auch zur Geschichte der bildenden Kunst äußerten. Von den Schriftstellern und Dichtern konnten nur ganz wenige berücksichtigt werden, die das Geschehen im Bereich der bildenden Kunst und die Vorstellungen von Kunstgeschichte maßgeblich und damit unübersehbar beeinflußten. Aus dem Bereich der "Künstlerästhetiken«, die vorzugsweise das eigene Schaffen kommentieren, war nur auf den Architekten Gottfried Semper mit seiner weitreichenden Stiltheorie nicht zu verzichten. Die große Zahl der mit der antiken Kunst befaßten Archäologen, von denen gewiß viele auch die Methoden auf dem Gebiet der "mittleren und neueren Kunstgeschichte" mitbestimmten, war nicht unterzubringen; für sie gibt es allerdings bereits entsprechende Nachschlagewerke. Winckelmann mußte aber seinen Platz in diesem Buch bekommen, weil er auch Begründer der Wissenschaft von nachantiker Kunst war. Um das Streben nach einer Weltgeschichte der Kunst, das vielen der interessantesten Kunsthistoriker am Herzen lag, wenigstens anzudeuten, wurden einige herausragende Spezialisten für einzelne Bereiche der älteren außereuropäischen und der frühgeschichtlichen Anfänge der Kunst behandelt. Insgesamt ist den Verfassern wohl bewußt, daß die hier vorgestellten Kunsthistoriker eine durchaus subjektive Auswahl bilden und es keine zwingenden Argumente gibt, warum viele fehlen, deren Leistungen für die international belangvolle Entwicklung der deutschen, österreichischen und schweizerischen Kunstwissenschaft um nichts geringer waren als die mancher ihrer Kollegen, die Berücksichtigung fanden.

Das Personenlexikon kann die Darstellung der Disziplingeschichte nicht ersetzen. Wir haben eine Auswahlbibliographic von zusammenfassenden wissenschaftsgeschichtlichen Arbeiten ergänzend hinzugefügt. Die Literaturhinweise zu den einzelnen Kunsthistorikern sind so ausgewählt, daß sie eine Vorstellung von deren Arbeitsgebieten und individueller Entwicklung vermitteln. Vorhandene Personalbibliographien werden verzeichnet. Neuauflagen und Übersetzungen in andere Sprachen sind nur angegeben, wenn sie besondere Schlüsse auf die Ausstrahlungskraft einer wissenschaftlichen Leistung erlauben. Rezensionen wurden unter dem gleichen Gesichtspunkt erfaßt. Der Widerhall, den Publikationen in den Arbeiten anderer Forscher fanden, konnte ebensowenig dokumentiert werden wie die Auseinandersetzung mit Person und Leistung eines Gelehrten in solchen Veröffentlichungen, die ihn nicht ausdrücklich in ihrem Titel erwähnen. Auch dazu darf auf die disziplingeschichtlichen Darstellungen verwiesen werden. Biographische Artikel in allgemeinen und fachspezifischen Lexika und in biographischen Sammelwerken werden aus Platzgründen nicht aufgelistet.

Beim Kunsthistorikerlexikon haben Kunsthistoriker aus drei Generationen und aus Berlin und Bochum zusammengearbeitet und in den Darstellungen ihres Interesses an der spannenden Geschichte des kunstwissenschaftlichen Denkens und Tuns viel Übereinstimmung festgestellt. Die Erarbeitung einiger Artikel übernahmen dankenswerterweise Dr. Karin Rührdanz (Halle) und Dr. Jürgen Zimmer (Berlin). Den freundlichen Mitarbeiterinnen und Mitarbeitern von Bibliotheken, in denen wir 
arbeiteten, den Fachkollegen, die uns wertvolle Hinweise gaben, und besonders unserem Lektor Dr. Oliver Schütze und Irene Kahlau danken wir aufrichtig. Wir hoffen auf eine produktive, kritische Benutzung unseres Lexikons, auf Nachfragen, Anregungen, auch Widerspruch von seiten möglichst vieler Kollegen, Studenten, Praktiker der Kunstvermittlung und anderer, die neugierig auf die Kunstgeschichte und die Protagonisten ihrer Erschließung sind.

Peter Betthausen, Peter H. Feist, Christiane Fork 
Artikel und Autoren

\begin{tabular}{|c|c|}
\hline Antal, Friedrich PHF & Friedlaender, Walter PHF \\
\hline Badt, Kurt PHF & Friedländer, Max Jacob Jz \\
\hline Bandmann, Günter PHF & Gall, Ernst $\quad \mathrm{CF}$ \\
\hline Bartsch, Adam von $\mathrm{CF}$ & Gantner, Joseph PB \\
\hline Bauch, Kurt Pв & Gerson, Horst PB \\
\hline Baum, Julius CF & Gerstenberg, Kurt PB \\
\hline Bayersdorfer, Adolf $\mathbf{P B}$ & Giedion, Sigfried PHF \\
\hline Beenken, Hermann PHF & Glaser, Curt CF \\
\hline Behne, Adolf PHF & Goethe, Johann Wolfgang von PB \\
\hline Benesch, Otto PHF & Goldschmidt, Adolph Рв \\
\hline Bezold, Gustav von CF & Gosebruch, Martin PHF \\
\hline Bloch, Peter PHF & Grimm, Herman PB \\
\hline Bode, Wilhelm von $\mathrm{PB}$ & Grisebach, August $\quad$ PB \\
\hline Boisserée, Sulpiz und Melchior $\mathrm{CF}$ & Gurlitt, Cornelius \\
\hline Braunfels, Wolfgang CF & Hagedorn, Christian Ludwig von \\
\hline Brinckmann, Albert Erich $\quad$ PB & Hagen, Ernst August CF \\
\hline Burckhardt, Jacob PB & Hager, Werner PB \\
\hline Burger, Fritz PHF & Hahnloser, Hans Robert PB \\
\hline Christ, Johann Friedrich $\mathrm{CF}$ & Hamann, Richard PHF \\
\hline Clasen, Karl-Heinz PHF & Hartlaub, Gustav Friedrich PHF \\
\hline Clemen, Paul jz & Haseloff, Arthur $\quad$ PB \\
\hline Dehio, Georg PB & Hausenstein, Wilhelm PHF \\
\hline Demus, Otto PHF & Hauser, Arnold PHF \\
\hline Diez, Ernst $\quad$ KR & Heidrich, Ernst $\quad P B$ \\
\hline Dohme, Robert $\mathrm{CF}$ & Heineken, Carl Heinrich von $\quad$ PB \\
\hline Drost, Willi PB & Heinse, Johann Jakob Wilhelm CF \\
\hline Dvořák, Max PHF & Heise, Carl Georg PB \\
\hline Eberlein, Kurt Karl PB & Hempel, Eberhard PB \\
\hline Einem, Herbert von PHF & Hentzen, Alfred PHF \\
\hline Einstein, Carl PHF & Herzfeld, Ernst $\quad$ KR \\
\hline Eitelberger, Rudolf von $\mathrm{PHF}$ & Hess, Walter CF \\
\hline Ettlinger, Leopold CF & Hetzer, Theodor PB \\
\hline Evers, Hans Gerhard CF & Heydenreich, Ludwig Heinrich \\
\hline Falke, Jakob von PHF & Hotho, Heinrich Gustav PB \\
\hline Fernow, Carl Ludwig $\mathrm{PB}$ & Imdahl, Max CF \\
\hline Feulner, Adolf $\mathrm{PB}$ & Jahn, Johannes PHF \\
\hline Fiedler, Konrad PB & Janitschek, Hubert PB \\
\hline Fiorillo, Johann Dominicus $\quad$ PB & Jantzen, Hans \\
\hline Förster, Ernst PB & Jordan, Max CF \\
\hline Fraenger, Wilhelm CF & Justi, Carl PB \\
\hline Frankl, Paul PHF & Justi, Ludwig PHF \\
\hline Frey, Dagobert PHF & Kallab, Wolfgang CF \\
\hline
\end{tabular}


Kauffmann, Hans PHF Kaufmann, Emil PHF

Kautzsch, Rudolf CF

Keller, Harald PB

Kinkel, Gottfried CF

Kohlhaussen, Heinrich CF

Kolloff, Eduard CF

Kraus, Franz Xaver CF

Krautheimer, Richard CF

Kugler, Franz Theodor PB

Kühn, Herbert PHF

Kühnel, Ernst $\quad$ KR

Ladendorf, Heinz PHF

Lehrs, Max Jz

Lichtwark, Alfred CF

Lippmann, Friedrich JZ

Löffler, Fritz PB

Lorck, Karl von PB

Lübke, Wilhelm PB

Lützeler, Heinrich $\mathrm{CF}$

Lützow, Karl von CF

Martin, Kurt CF

Mayer, August Liebmann $\quad \mathrm{CF}$

Meder, Joseph CF

Meier-Graefe, Julius PrfF

Merck, Johann Heinrich CF

Metz, Peter CF

Meyer, Erich CF

Meyer, Johann Heinrich PB

Meyer, Julius CF

Morelli, Giovanni PB

Muther, Richard PHF

Neumann, Karl PB

Neumeyer, Alfred PHF

Novotny, Fritz PHF

Osten, Gert von der CF

Paatz, Walter PB

Pächt, Otto PHF

Panofsky, Erwin PB

Passavant, Johann David PB

Pauli, Gustav PHF

Pecht, Friedrich PHF

Pevsner, Sir Nikolaus PHF

Pinder, Wilhelm PB

Raczynski, Athanasius Graf PB
Raphael, Max PHF

Rave, Paul Ortwin CF

Reidemeister, Leopold CF

Riegel, Herman CF

Riegl, Alois PHF

Rintelen, Friedrich PB

Rosenberg, Adolf PHF

Rosenberg, Jakob CF

Rumohr, Karl Friedrich von PB

Sandrart, Joachim von PB

Sauerlandt, Max CF

Saxl, Fritz CF

Schardt, Alois PHF

Scheffler, Karl PHF

Schlegel, August Wilhelm und Friedrich PB

Schlosser, Julius Ritter von PHF

Schmalenbach, Fritz PHF

Schmarsow, August PB

Schmid, Heinrich Alfred CF

Schmidt, Georg PHF

Schmitt, Otto CF

Schnaase, Karl PB

Schorn, Ludwig PB

Schrade, Hubert PB

Schubring, Paul CF

Schürer, Oskar PHF

Sedlmayr, Hans PHF

Seidlitz, Woldemar von PB

Semper, Gottfried PB

Simson, Otto von $\mathrm{CF}$

Singer, Hans Wolfgang PB

Springer, Anton PB

Stange, Alfred PB

Stechow, Wolfgang PB

Strzygowski, Josef PHF

Swarzenski, Georg PB

Swarzenski, Hanns PB

Swoboda, Karl Maria PHF

Thausing, Moriz PHF

Thieme, Ulrich CF

Thode, Henry PB

Tietze, Hans PHF

Tintelnot, Hans PHF

Tschudi, Hugo von PHF

Vischer, Robert $\mathrm{PB}$ 
Vitzthum, Georg Graf PB Vöge, Wilhelm pв

Volbach, Wolfgang Fritz PHF

Voss, Hermann PB

Waagen, Gustav Friedrich PB

Wackenroder, Wilhelm Heinrich

Wackernagel, Martin PB

Waetzoldt, Wilhelm PB

Wagner-Rieger, Renate PHF

Waldmann, Emil PHF

Warburg, Aby CF

Weigert, Hans PB

Weisbach, Werner PHF
Weise, Georg CF

Westheim, Paul PHF

Wickhoff, Franz PHF

Wilpert, Josef CF

Winckelmann, Johann Joachim CF

Wind, Edgar $\mathrm{CF}$

Winkler, Friedrich JZ

Wittkower, Rudolf PHF

Woermann, Karl PB

Wölfflin, Heinrich PB

Woltmann, Alfred PB

Worringer, Wilhelm PHF

Wulff, Oskar PHF 


\section{Abkürzungen}

I. Orte

$\begin{array}{llll}\text { Au } & \text { Augsburg } & \text { Kr } & \text { Karlsruhe } \\ \text { BB } & \text { Baden-Baden } & \text { Lo } & \text { London } \\ \text { Bie } & \text { Bielefeld } & \text { Lpz } & \text { Leipzig } \\ \text { Bln } & \text { Berlin } & \text { Lü } & \text { Lübeck } \\ \text { Br } & \text { Breslau } & \text { Mar } & \text { Marburg/Lahn } \\ \text { Bschw } & \text { Braunschweig } & \text { Mh } & \text { Mannheim } \\ \text { Cam } & \text { Cambridge } & \text { Mü } & \text { München } \\ \text { Ch } & \text { Chicago } & \text { Nü } & \text { Nürnberg } \\ \text { Da } & \text { Darmstadt } & \text { NY } & \text { New York } \\ \text { Dr } & \text { Dresden } & \text { Ox } & \text { Oxford } \\ \text { Dü } & \text { Düsseldorf } & \text { Pad } & \text { Paderborn } \\ \text { Fl } & \text { Florenz } & \text { Pd } & \text { Potsdam } \\ \text { FrB } & \text { Freiburg i.Br. } & \text { Phi } & \text { Philadelphia } \\ \text { Frf } & \text { Frankfurt/Main } & \text { Ra } & \text { Ravensburg } \\ \text { Gö } & \text { Göttingen } & \text { Sa } & \text { Salzburg } \\ \text { Gü } & \text { Gütersloh } & \text { St } & \text { Stettin } \\ \text { Ha } & \text { Hannover } & \text { Ste } & \text { Stendal } \\ \text { HaS } & \text { Halle/Saale } & \text { Stg } & \text { Stuttgart } \\ \text { Hbg } & \text { Hamburg } & \text { Str } & \text { Straßburg, Strasbourg } \\ \text { Hei } & \text { Heidelberg } & \text { Tü } & \text { Tübingen } \\ \text { Hil } & \text { Hildesheim } & \text { Wa } & \text { Washington } \\ \text { Ka } & \text { Kassel } & \text { Wb } & \text { Wiesbaden } \\ \text { Kö } & \text { Königsberg } & \text { Wei } & \text { Weimar } \\ \text { KöT } & \text { Königstein im Taunus } & \text { Zü } & \text { Zürich } \\ & & & \\ & & & \end{array}$

2. Zeitschriften, Periodika, Sammelbände

$\begin{array}{lll}\text { ABKK } & \begin{array}{l}\text { Amtliche Berichte aus } \\ \text { den kgl. Kunstsammlun- }\end{array} & \text { ACIB } \\ & \text { gen (Berlin) } \\ \text { ACA } & \text { Atti del I Convegno } \\ & \text { sulle arti minori in To- } \\ & \text { scana. Arezzo r971, Flo- } \\ & \text { renz I973 } \\ & \text { Actes du XVIIe Congrès } \\ \text { ACIA } & \text { International d' histoire } \\ & \text { de l' art. Amsterdam } \\ & \text { I952, Den Haag I955 }\end{array}$

Atti del XXIV Congresso Internazionale di Storia dell' Arte. Bologna I979, Bologna I $982-84$ Il Vasari storiografo e artista. Atti del Congresso Internazionale nel IV Centenario della morte. Arezzo/Florenz 1974, Florenz 1976 


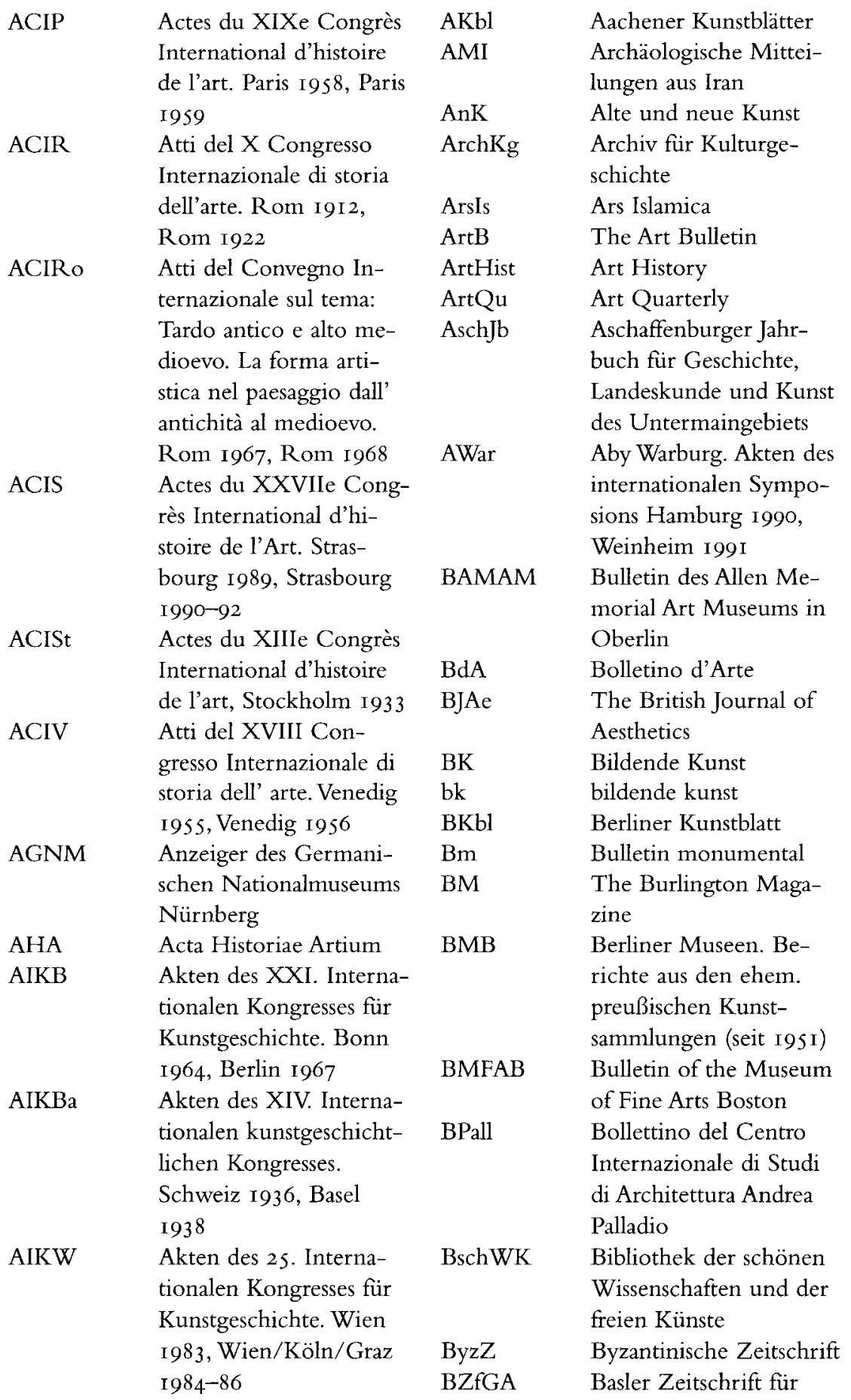


Geschichte und Alter-

tumskunde

$\begin{array}{ll}\text { CArtJ } & \text { College Art Journal } \\ \mathrm{CdA} & \text { Critica d'Arte } \\ \mathrm{Ci} & \text { Der Cicerone }\end{array}$

DJbVk Deutsches Jahrbuch für

Volkskunde

DKbl Das Kunstblatt

DKDpf Deutsche Kunst und

Denkmalpflege

DKuD Deutsche Kunst und

Dekoration

DLZ Deutsche Literaturzeitung

Dpfl Die Denkmalpflege

DtKbl Deutsches Kunstblatt

DVjS Deutsche Vierteljahrs-

schrift für Literaturwis-

senschaft und Geistesge-

schichte

DWdK Das Werk des Künstlers

EGA

Erbe als Gegenwartsauf-

gabe. Referate der Ar-

beitstagung des Bereichs

Kunstwissenschaft der

Humboldt-Universität

I6.-I 8. 4. I975, 2 Teile,

$B \ln 1975$

FuB Forschungen und Berichte. Staatliche $\mathrm{Mu}-$ seen zu Berlin

FuF Forschungen und Fortschritte. Nachrichtenblatt der deutschen Wissenschaft und Technik

GBA Gazette des Beaux Arts

GöGA Göttingische gelehrte

Anzeigen

GrK Die graphischen Künste

GRM Germanisch-romanische

Monatsschrift

Heijbb Heidelbergische Jahrbücher

HJbGG Historisches Jahrbuch

der Görres-Gesellschaft
HkSUMü

$\mathrm{HZ}$

JAAC

$\mathrm{Jb} \ddot{\mathrm{AaK}}$

JbAdW

JbAsKunst

JbBAdW

JbbdG

JbBM

JbDAI

$\mathrm{JbFDH}$

JbGHK

JbGoe

JbHK

JbHKh

JbhVk

JbKDr

JbkSAK

JbkSW
Hefte des kunsthistorischen Seminars der Universität München

Historische Zeitschrift

The Journal of Aesthetics and Art Criticism

Jahrbuch für Ästhetik und allgemeine Kunstwissenschaft

Jahrbuch der Deutschen

Akademie der Wissen-

schaften zu Berlin

Jahrbuch der Asiatischen

Kunst

Jahrbuch der Bayeri-

schen Akademie der

Wissenschaften.

Jahrbücher der deut-

schen Geschichte

Jahrbuch der Berliner

Museen

Jahrbuch des Deutschen

Archäologischen Instituts

Jahrbuch des Freien

Deutschen Hochstifts

Jahrbuch der Gesellschaft

Hamburgischer Kunst-

freunde

Jahrbuch der Goethe-

Gesellschaft

Jahrbuch der Hamburger

Kunstsammlungen

Idea. Jahrbuch der Hamburger Kunsthalle

Jahrbuch für historische

Volkskunde

Jahrbuch der Staatlichen

Kunstsammlungen

Dresden

Jahrbuch der kunsthistorischen Sammlungen des

Allerhöchsten Kaiserhauses

Jahrbuch der kunsthistorischen Sammlungen in Wien 


\begin{tabular}{|c|c|c|c|}
\hline JbKw & $\begin{array}{l}\text { Jahrbuch für Kunstwis- } \\
\text { senschaft }\end{array}$ & & $\begin{array}{l}\text { bureau voor kunsthisto- } \\
\text { rische Documentatie }\end{array}$ \\
\hline JbLiturW & $\begin{array}{l}\text { Jahrbuch für Liturgie- } \\
\text { wissenschaft }\end{array}$ & $\mathrm{KschH}$ & $\begin{array}{l}\text { Die Kunst und das } \\
\text { schöne Heim }\end{array}$ \\
\hline \multirow[t]{3}{*}{ JbPK } & Jahrbuch der Königl. & Ktid & Konsthistorisk tidskrift \\
\hline & Preußischen Kunst- & KtKtler & Kunst und Künstler \\
\hline & sammlungen & Ktw & Das Kunstwerk \\
\hline JbPKB & Jahrbuch der Stiftung & $\mathrm{KuOr}$ & Kunst des Orients \\
\hline & $\begin{array}{l}\text { Preußischer Kulturbesitz } \\
\text { Jahrbuch der Ruhr-Uni- }\end{array}$ & LZB & $\begin{array}{l}\text { Literarisches Zentralblatt } \\
\text { für Deutschland }\end{array}$ \\
\hline JbRUB & versität Bochum & Marjb & Marburger Jahrbuch für \\
\hline \multirow[t]{2}{*}{$\mathrm{JbSchIKw}$} & Jahrbuch des Schweize- & & Kunstwissenschaft \\
\hline & $\begin{array}{l}\text { rischen Instituts für } \\
\text { Kunstwissenschaft }\end{array}$ & MDAI & $\begin{array}{l}\text { Mitteilungen des Deut- } \\
\text { schen Archäologischen }\end{array}$ \\
\hline \multirow[t]{2}{*}{$\mathrm{JbZIfKg}$} & Jahrbuch des Zentralin- & & Instituts \\
\hline & stituts für Kunstge- & $\mathrm{MdG}$ & Museum der Gegenwart \\
\hline \multirow[t]{2}{*}{$\mathrm{JbZk}$} & $\begin{array}{l}\text { schichte } \\
\text { Jahrbuch der k.k. Zen- }\end{array}$ & $\mathrm{MfKw}$ & $\begin{array}{l}\text { Monatshefte für Kunst- } \\
\text { wissenschaft }\end{array}$ \\
\hline & $\begin{array}{l}\text { tralkommission zur Er- } \\
\text { forschung und Erhaltung } \\
\text { der Baudenkmale }\end{array}$ & MGNM & $\begin{array}{l}\text { Mitteilungen aus dem } \\
\text { Germanischen National- } \\
\text { museum Nürnberg }\end{array}$ \\
\hline JSAH & $\begin{array}{l}\text { Journal of the Society of } \\
\text { Architectural Historians }\end{array}$ & MGvK & $\begin{array}{l}\text { Mitteilungen der Gesell- } \\
\text { schaft für vergleichende }\end{array}$ \\
\hline JWCI & Journal of the Warburg & & Kunstforschung \\
\hline \multirow[t]{3}{*}{ KA } & $\begin{array}{l}\text { and Courtauld Institutes } \\
\text { Kunstgeschichtlicher An- } \\
\text { zeiger (Beihefte zu den }\end{array}$ & MIfÖG & $\begin{array}{l}\text { Mitteilungen des Insti- } \\
\text { tuts für Österreichische } \\
\text { Geschichtsforschung }\end{array}$ \\
\hline & $\begin{array}{l}\text { Mitteilungen des Insti- } \\
\text { tuts für Österr. Ge- }\end{array}$ & MJbbK & $\begin{array}{l}\text { Münchner Jahrbuch der } \\
\text { bildenden Kunst }\end{array}$ \\
\hline & schichtsforschung) & MKhIF & Mitteilungen des Kunst- \\
\hline $\mathrm{KB}$ & Kritische Berichte & & historischen Instituts in \\
\hline \multirow[t]{2}{*}{$\mathrm{Kbl}$} & Kunstblatt. Morgenblatt & & Florenz \\
\hline & für gebildete Stände & $\mathrm{MZk}$ & Mitteilungen der k. k. \\
\hline KBLit & $\begin{array}{l}\text { Kritische Berichte zur } \\
\text { kunstgeschichtlichen Li- } \\
\text { teratur }\end{array}$ & & $\begin{array}{l}\text { Zentralkommission zur } \\
\text { Erforschung und Erhal- } \\
\text { tung der Baudenkmale }\end{array}$ \\
\hline KChr & Kunstchronik & MÖMKI & Mitteilungen des k.k. \\
\hline $\mathrm{KF}$ & $\begin{array}{l}\text { Kunstwissenschafliche } \\
\text { Forschungen }\end{array}$ & & $\begin{array}{l}\text { österreichischen Muse- } \\
\text { ums für Kunst und In- }\end{array}$ \\
\hline $\mathrm{KfA}$ & Kunst für Alle & & dustrie \\
\hline \multirow[t]{2}{*}{$\mathrm{KJbBH}$} & Kunstgeschichtliches & MusJ & Museumsjournal \\
\hline & $\begin{array}{l}\text { Jahrbuch der Bibliotheca } \\
\text { Hertziana }\end{array}$ & $\mathrm{NdtBKg}$ & $\begin{array}{l}\text { Niederdeutsche Beiträge } \\
\text { zur Kunstgeschichte }\end{array}$ \\
\hline KMRB & $\begin{array}{l}\text { Kunsthistorische Mede- } \\
\text { delingen van het Rijks- }\end{array}$ & NHeijbb & $\begin{array}{l}\text { Neue Heidelberger Jahr- } \\
\text { bücher }\end{array}$ \\
\hline
\end{tabular}




\begin{tabular}{|c|c|c|c|}
\hline $\mathrm{NKJb}$ & $\begin{array}{l}\text { Nederlands Kunsthisto- } \\
\text { risch Jaarboek }\end{array}$ & & $\begin{array}{l}\text { schrift der Universität } \\
\text { Leipzig }\end{array}$ \\
\hline $\mathrm{OH}$ & Oud Holland & \multirow[t]{3}{*}{ ZDMG } & Zeitschrift der Deut- \\
\hline OMD & Old Master Drawings & & schen Morgenländischen \\
\hline \multirow[t]{3}{*}{ ÖZKD } & Österreichische Zeit- & & Gesellschaft \\
\hline & schrift für Kunst und & \multirow[t]{2}{*}{ ZDVKw } & Zeitschrift des deutschen \\
\hline & Denkmalpflege & & Vereins für Kunstwissen- \\
\hline $\mathrm{PhJb}$ & Philosophisches Jahrbuch & & schaft \\
\hline PJbb & Preußische Jahrbücher & \multirow[t]{3}{*}{ ZfÄaK } & Zeitschrift für Ästhetik \\
\hline RA & Revue de l'Art & & und allgemeine Kunst- \\
\hline \multirow[t]{2}{*}{$\mathrm{RACr}$} & Rivista di Archeologia & & wissenschaft \\
\hline & Cristiana & \multirow[t]{2}{*}{$\mathrm{ZfBf}$} & Zeitschrift für Bücher- \\
\hline $\mathrm{RdA}$ & Rivista d'Arte & & freunde \\
\hline $\mathrm{RfKw}$ & $\begin{array}{l}\text { Repertorium für Kunst- } \\
\text { wissenschaft }\end{array}$ & $\mathrm{ZfbK}$ & $\begin{array}{l}\text { Zeitschrift für bildende } \\
\text { Kunst }\end{array}$ \\
\hline \multirow[t]{2}{*}{ RJbKg } & Römisches Jahrbuch für & ZfBw & Zeitschrift für Bauwesen \\
\hline & Kunstgeschichte & \multirow[t]{2}{*}{$\mathrm{ZfchK}$} & Zeitschrift für christliche \\
\hline \multirow[t]{2}{*}{ SberKgG } & Sitzungsberichte. Kunst- & & Kunst \\
\hline & $\begin{array}{l}\text { geschichtliche Gesell- } \\
\text { schaft Berlin }\end{array}$ & $\mathrm{ZfdB}$ & $\begin{array}{l}\text { Zeitschrift für deutsche } \\
\text { Bildung }\end{array}$ \\
\hline StG & Studium Generale & \multirow[t]{2}{*}{ ZfdGw } & Zeitschrift für deutsche \\
\hline $\mathrm{StJb}$ & Städel-Jahrbuch & & Geisteswissenschaft \\
\hline SvLitg & $\begin{array}{l}\text { Studien zur vergleichen- } \\
\text { den Literaturgeschichte }\end{array}$ & ZfdKph & $\begin{array}{l}\text { Zeitschrift für deutsche } \\
\text { Kulturphilosophie }\end{array}$ \\
\hline TM & Der Teutsche Merkur & $\mathrm{ZfK}$ & Zeitschrift für Kunst \\
\hline UKd & Unsere Kunstdenkmäler & \multirow[t]{2}{*}{$\mathrm{ZfKg}$} & Zeitschrift für Kunstge- \\
\hline VBW & Vorträge der Bibliothek & & schichte \\
\hline \multirow{3}{*}{ VSWG } & Warburg & \multirow[t]{2}{*}{$\mathrm{ZfKw}$} & Zeitschrift für Kunstwis- \\
\hline & Vierteljahrschrift für So- & & senschaft \\
\hline & $\begin{array}{l}\text { zial- und Wirtschaftsge- } \\
\text { schichte }\end{array}$ & \multirow[t]{2}{*}{ ZfPhK } & $\begin{array}{l}\text { Zeitschrift für Philoso- } \\
\text { phie und philosophische }\end{array}$ \\
\hline WB & Weimarer Beiträge & & Kritik \\
\hline WJbfKg & $\begin{array}{l}\text { Wiener Jahrbuch für } \\
\text { Kunstgeschichte }\end{array}$ & $\mathrm{ZfRGg}$ & $\begin{array}{l}\text { Zeitschrift für Religi- } \\
\text { ons- und Geistesge- }\end{array}$ \\
\hline \multirow[t]{2}{*}{ WMh } & Westermanns illustrierte & \multirow{3}{*}{ ZSchAK } & schichte \\
\hline & deutsche Monatshefte & & Zeitschrift fuir Schweize- \\
\hline WRJb & $\begin{array}{l}\text { Wallraf-Richartz-Jahr- } \\
\text { buch }\end{array}$ & & $\begin{array}{l}\text { rische Archäologie u. } \\
\text { Kunstgeschichte }\end{array}$ \\
\hline \multirow[t]{3}{*}{ WZHU } & Wissenschaftliche Zeit- & \multirow{5}{*}{$\begin{array}{l}\text { Altmeister } \\
\text { I } 990\end{array}$} & \\
\hline & schrift der Humboldt- & & Dilly, Heinrich (Hrsg.): \\
\hline & Universität Berlin & & Altmeister moderner \\
\hline WZUH & Wissenschaftliche Zeit- & & Kunstgeschichte, Berlin \\
\hline & schrift der Universität & & I990 \\
\hline & $\begin{array}{l}\text { Halle } \\
\text { Wissenschaftliche Zeit- }\end{array}$ & $\begin{array}{l}\text { Archäologen- } \\
\text { bildnisse I } 988\end{array}$ & $\begin{array}{l}\text { Lullies, Reinhard/Schie- } \\
\text { ring, Wolfgang (Hrsg.): }\end{array}$ \\
\hline
\end{tabular}


Archäologenbildnisse.

Porträts und Kurzbiographien von Klassischen

Archäologen deutscher

Sprache, Mainz I99I (2. Aufl.)

Hüttinger Hüttinger, Eduard: Por-

1992

Jahn 1924 träts und Profile. Zur Geschichte der Kunstgeschichte, St. Gallen 1992 Jahn, Johannes (Hrsg.): Die Kunstwissenschaft der Gegenwart in Selbst- darstellungen, Leipzig I 924

Sitt 1990 Sitt, Martina (Hrsg.):

Kunsthistoriker in eigener Sache, Berlin I990

Waetzoldt Waetzoldt, Wilhelm:

I92 I, I924 Deutsche Kunsthistoriker. Bd. I: Von Sandrart bis Rumohr, Leipzig I92 I; Bd. 2: Von Passavant bis Justi, Leipzig 1924

\section{Sonstiges}

\begin{tabular}{|c|c|c|c|}
\hline Abh. & Abhandlung & Hrsg., hrsg. & $\begin{array}{l}\text { Herausgeber, herausge- } \\
\text { geben }\end{array}$ \\
\hline AdW & $\begin{array}{l}\text { Akademie der Wissen- } \\
\text { schaften }\end{array}$ & Inst. & $\begin{array}{l}\text { geben } \\
\text { Institut }\end{array}$ \\
\hline Akad. & Akademie & internat. & international \\
\hline AKat. & Ausstellungskatalog & ital. & italienisch \\
\hline Anh. & Anhang & Jb., Jbb. & Jahrbuch, Jahrbücher \\
\hline Anz. & Anzeiger & Jh. & Jahrhundert \\
\hline a. o. & außerordentlicher/s & Kat. & Katalog \\
\hline & (Professor, Mitglied) & kgl. & königlich \\
\hline Ber. & Bericht(e) & Kgr. & Königreich \\
\hline Bibl. & Bibliothek & $\mathrm{Kl}$. & Klasse \\
\hline Bibliogr. & Bibliographie & Mitt. & Mitteilung(en) \\
\hline Diss. & Dissertation & $\mathrm{Nd}$. & Neudruck \\
\hline dt. & deutsch & ndld. & niederländisch \\
\hline Einl. & Einleitung & o. & ordentlicher/s (Professor \\
\hline engl. & englisch & & Mitglied) \\
\hline Erg. & Ergänzung & o. $\mathrm{O}$. & ohne Ort \\
\hline $\mathrm{F}$ & Folge & österr. & österreichisch \\
\hline frz. & französisch & phil. & philosophisch \\
\hline FS & Festschrift & $\mathrm{R}$. & Reihe \\
\hline Ges. & Gesellschaft & Res. & Resumé \\
\hline ges.wiss. & gesellschaftswissenschaft- & Rez. & Rezension \\
\hline & lich & Sber. & Sitzungsbericht(e) \\
\hline Gesch. & Geschichte & Slg. & Sammlung \\
\hline $\mathrm{H}$. & Heft & Sp. & Spalte \\
\hline $\mathrm{Hdb}$. & Handbuch & u. Z. & unserer Zeitrechnung \\
\hline hist. & historisch & Univ. & Universität \\
\hline
\end{tabular}


v. u. Z.

Verf.

Veröff.

Verz.

4. Autoren

PB

PHF

$\mathrm{CF}$ vor unserer Zeitrechnung

Verfasser

Veröffentlichung

Verzeichnis
Vorw.

Wiss.

Ztschr.
Vorwort

Wissenschaft, wissenschaftlich

Zeitschrift

KR

Karin Rührdanz

JZ

Jürgen Zimmer

Peter H. Feist

Christiane Fork 